\title{
The Role of MRI in Diagnosis and Management of Breast Cancer During Fertility Period
}

\author{
Medhat M. Refaat, Ahmed A. Abou El-Soud, Manar Ahmed
}

\begin{abstract}
Department of diagnostic and interventional radiology, Benha faculty of medicine, Banha University, Egypt.

Correspondence to: Manar Ahmed, Department of diagnostic and interventional radiology, Benha faculty of medicine, Banha University, Egypt.
\end{abstract}

\section{Email:}

dr_manora@windowslive.com

Received: 8 November 2019

Accepted: 11 December 2021

\begin{abstract}
:
Background: Breast MRI is no longer considered as an experimental modality, but has reached a solid position in the diagnosis and workup of breast lesions. Aim:is to assess the impact of contrast enhanced MRI in the diagnosis and management of breast cancer in young female patients. Patients \& methods: Retrospective descriptive study of imaging findings of pathologically proved breast cancer in young female patients, from Kasr Al Ainy hospital, National cancer institute and El Sahel teaching hospital. From Jan.2018 to June 2018. The patients were referred from general surgery department. Approval of the local ethical committee were taken in consideration. Economic status of the patients have been considered.25 patients, Ages ranged between 20 and 40 years (mean age 30); the previous ultrasound and mammography scans done for the patients to assess the actual size, metabolic behaviour of the lesion and its relation to the surrounding structures. Results: The current study showed that 18/25(72\%) cases
\end{abstract} had multicentric breast cancer and 7/25 (28\%) cases had single masses. 21/25 (84\%) cases were missed by mammography. 14/25 (56\%) cases were missed by ultrasound. Conclusion: We concluded that DCE-MRI has a vital role in the diagnostic work up and management of breast cancer in young women. MRI revealed most specific criteria for malignancy and it has a great ability to detect both of main lesion and additional lesions(multicentric malignancy).MRI is a very important modality as it can assist in management by more accurate assessment of the extent of disease.

Key words: MRI, Breast cancer, fertility.

\section{Abbreviations:}

MRI: Magnetic resonance imaging, DCE: dynamic contrast enhanced, US: ultrasound. 


\section{Introduction:}

Breast cancer in young women is a relatively uncommon condition but it accounts for more than $40 \%$ of all incidental female cancer cases in these women $^{(1)}$

In women of high risk for breast cancer, mammographic screening has shown a low sensitivity (29-50\%) compared with that of the screening done for the general female population (70-80\%), with a higher rate of nodal involvement (20-56\% versus $22 \%)$ and higher percentages of interval cancers (35-50\% versus $20-25 \%)^{(2)}$

MRI may be predominantly helpful in certain situations. This includes high risk cases especially those who have dense breast tissue. Dense breast tissue in young women may obscure signs of malignancy on mammography and limits the evaluation of the true extent of disease. ${ }^{(3)}$

MRI is emerging as a very important modality to assess response of the disease to the neo-adjuvant therapy, not only because it can define the extent of disease and accurately evaluate response to therapy, but also as it enables us to look at the morphology of tumors and identify tumor patterns that are different at initial presentation. ${ }^{(3)}$

\section{Patient and methods:}

1. Patients Retrospective descriptive analysis of the imaging findings of pathologically proved breast cancer in young female patients. ${ }^{5}$ patients radiologically proved to have breast cancer, referred to radiology department from outpatient clinic surgery of Kasr Al Ainy hospital, National cancer institute and $\mathrm{El}$ Sahel teaching hospital. The patients' age ranged between 20 and 40 years (median 30).

Inclusion Criteria: female patient aged from 20 to 40 years old proved to have breast cancer and candidate for MRI examination.

Exclusion Criteria: Patients having a contraindication to MRI.

\section{Methods}

The patients were subjected to clinical history taking, clinical examination, mammography, breast ultrasonography and dynamic contrast MRI.

Approval of the local ethical committee were taken in consideration. The patient was aware of the examination, economic status of the patient have been considered 
and the patient has to get benefit from the examination.

\section{Dynamic Contrast Enhanced MRI}

\section{Protocol:}

Patients leave metallic objects at home or remove them before your MRI and breathe normally but to lie as still as possible. Patient lies prone with breast positioned in double breast coil

Dynamic contrast enhanced MRI was performed to all patients, pre-contrast axial T1, axial STIR, axial T2, DWI, ADC, sagittal T2 and post contrast axial 3D Dynamic, sagittal 3D T1 DIXON and kinetic curve assessment. All patients were injected Gd-containing contrast agent in the antecubital vein via $20 \mathrm{G}$ or $22 \mathrm{G}$ intravenous cannula.

\section{Findings that were detected by the}

\section{examination:}

- The lesion size, multiplicity and relation to surrounding vascular and soft tissue structures.

- Pattern of lesions enhancement and their metabolic activity.

- Presence or absence of pathologically enlarged axillary lymph nodes.

\section{○ Statistical Analysis}

- The collected data were organized, tabulated and statistically analyzed.

\section{$\underline{\text { Results: }}$}

- The patient's age ranged between 20 and 40 years (median 30). Patients' sex: females $(100 \%)$.

- The study is a retrospective descriptive analysis of the imaging findings of radiologically proved breast cancer in young patients.

- 18/25 (72\%) cases had multicentric breast cancer (2 patients $(8 \%)$ had bilateral multicentric breast cancer, 16/25 (64\%) cases had unilateral multicentric BC). 7/25 (28\%) cases had single masses.

- 19/25 (76\%) cases presented with palpable mass lesions and 6/25 (24\%) cases presented with mastitis.

- $5 / 25(20 \%)$ cases gave positive family history $(\mathrm{FH})$ of breast cancer and 20/25 (80\%) cases gave no family history.

- 4/25 (16\%) cases were detected and diagnosed by mammography and $21 / 25(84 \%)$ cases were missed by mammography.

- 11/25 (44\%) cases were detected and diagnosed by ultrasound and 14/25 $(56 \%)$ cases were missed by ultrasound. 
- DCE-MRI findings: 17/25 (68\%) cases had pure mass lesions, 1/25 (4\%) cases had pure non-mass lesion, 7/25 (28\%) cases had mixed lesions (6 cases had mass and non-mass lesions, 1 case had mixed mass and focus).

- Associated Findings: 5/25 (20\%) cases had associated edema, 3/25 (12\%) cases had skin thickening, 1/25 (4\%) case had nipple retraction, 2/25 (8\%) cases had skin dimpling.

- Lesion multiplicity: 18/25 (72\%) cases had multicentric breast cancer, 7/25 (28\%) cases had single masses, 11/25 (44\%) cases had single masses on ultrasound and proved multicentric on MRI.

- Post NAC \& pre-operative MRI changed management of: 19/25 (76\%) cases had mastectomy, 6/25 (24\%) cases had conservative surgery.

\section{Discussion:}

Many breast manifestations in young women are misdiagnosed by clinicians and radiologists and reported as cysts or benign breast lesions and they usually choose a 'follow up' protocol (3).

It is important to consider the possibilities of a malignancy in symptomatic young females despite the low prevalence of breast cancer in this group. This was evident in our study where most patients (76\% of cases) presented with a large palpable lump due to the delayed presentation and $24 \%$ presented with mastitis. This finding is compared with the study conducted by before ${ }^{(4)}$, in which 78.4 $\%$ of their cases had palpable masses and $3 \%$ presented with mastitis.

In our study $20 \%$ of patients gave positive family history in comparison with the study conducted by before ${ }^{(4)}$, there was a family history of breast cancer for $39.5 \%$ of cases. But in $2014^{(1)}$ study, only $5.1 \%$ of the cases had positive family history.

In $2014^{{ }^{(1)}}$ study dense parenchyma (ACR c or ACR d) was found in only $13 \%$ of the cases, while in our study it was found in $84 \%$ of the cases. In $2013^{(4)}$ study, $73.2 \%$ of their patients the parenchymal density was ACR c or ACR d, which agreed more with our study results.

In our study, mammography diagnosed only $16 \%$ of the patients and the false negative patients were $84 \%$. Of the 21 $(84 \%)$ false negatives, $17(81 \%)$ cases showed heterogeneously to extremely dense breast tissue composition on mammography. In contrary in 2014 ( ${ }^{1)}$ study reported that $87.4 \%$ of patients were visible on mammography and the false 
negative rate was $12.6 \%$. Of the 12 false negatives, 11 patients showed heterogeneously to extremely dense breast tissue composition on mammography.

As multicentric malignancy is of high incidence in young women, MRI has an important role due to its ability to detect both of the main lesion and additional lesions which is $100 \%$. This was evident in our study as all multicentric malignancies were detected by MRI.

In our study the multicentric malignancies were detected in $72 \%$ of the patients. Our results agreed with $2014^{(1)}$ study, where multicentric malignancies found in $75.9 \%$. In An et al, 2014 study, on MRI, the most frequent abnormalities were masses in 82.8 $\%$ of the patients. In our study $68 \%$ of the patients had masses. The most common findings were heterogeneous enhancing, irregular masses with irregular borders, and early rapid enhancement and delayed washout. The average size of masses detected and measured by MRI was $6 \mathrm{~cm}$.

Once a patient has been diagnosed with breast cancer, MRI can assist in patient management by the accurate assessment of the disease extent. MRI may be useful in predicting response and determining at an early time point a patient's response to treatment.
In $2012^{(5)}$ study reported that results of 216 women with locally advanced breast cancer underwent neo-adjuvant chemotherapy determined MRI findings were strong predictors of pathological response to neoadjuvant chemotherapy. In $2005^{(6)}$ study, on MRI, $74 \%$ of the patients showed response, and only $26 \%$ of the patients showed no response (had stationary course of the disease).

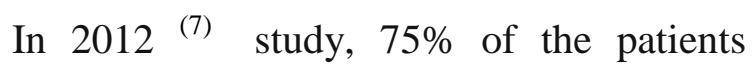
treated with mastectomy after MRI for so many reasons as $3.6 \%$ of the patients had additional cancers detected in the contralateral breast by MRI alone while there were additional malignant lesions identified in the ipsilateral breast $(13.7 \%$ of the patients) by MRI. The added cancer detection is associated with an impact on surgical decision making. While $25 \%$ of them received a conservative surgery and their study reported that, patients assessed by breast MRI following neo-adjuvant therapy and before surgery had an excellent outcome regardless the type of surgery (mastectomy or quadrantectomy).

In $2003^{(8)}$ study, $81 \%$ of the patients were surgically treated with MRM, and 19\% (4 patients) underwent breast conservation surgery. Of the patients who opted for breast conservation surgery, three had 
resectable tumor with negative surgical margins after the initial surgery, and only one had positive surgical margins that required further surgical intervention. None of the four women who underwent breast conservative surgery ultimately underwent mastectomy. While in $2005^{(6)}$ study, $58 \%$ of the patients had breast conservative surgery, while $42 \%$ had mastectomy as the final surgery.

Our study showed that the accuracy of MRI was $100 \%$ for the detection and characterization of breast cancers as in a previous study (4) study, where MRI identified all cancers. The accuracy of the ultrasonography in our study was $44 \%$.

\section{Conclusion:}

MRI is a very important modality as it can assist in management by more accurate assessment of the extent of disease and the response to neo-adjuvant chemotherapy. MRI is proved in our study to be useful in predicting response through defining the course of disease.

Our study showed that MRI in pre-surgery setting can modify treatment decisions. We concluded that DCE-MRI has a vital role in the diagnostic work up and management of breast cancer in young women. It seems probably no longer ethical to offer film mammograms to young women if MRI is feasible.

\section{References:}

1- An, Y. Y., Kim, S. H., \& Kang, B. J. (2014). Characteristic features and usefulness of MRI in breast cancer in patients under 40 years old: correlations with conventional imaging and prognostic factors. Breast Cancer, 21(3), 302-315.

2- Sardanelli, F., Santoro, F., \& Podo, F. (2016). Screening high risk women with MRI alone? European journal of radiology, 81, S137S138.

3- Salem, D. S., Kamal, R. M., Mansour, S. M., Salah, L. A., \& Wessam, R. (2013). Breast imaging in the young: the role of magnetic resonance imaging in breast cancer screening, diagnosis and follow-up. Journal of thoracic disease, 5(Suppl 1), S9.

4- Bullier, B., MacGrogan, G., Bonnefoi, H., Hurtevent-Labrot, G., Lhomme, E., Brouste, V. et al (2013). Imaging features of sporadic breast cancer in women under 40 years old: 97 cases. European radiology, 23(12), 32373245.

5- Lehman, C. D. (2012). Clinical indications: what is the evidence?. European journal of radiology, 81, S82-S84.

6- Yeh, E., Slanetz, P., Kopans, D. B., Rafferty, E., Georgian-Smith, D., Moy, L. et al (2005). Prospective comparison of mammography, sonography, and MRI in patients undergoing neoadjuvant chemotherapy for palpable breast cancer. American Journal of Roentgenology, 184(3), 868-877. 
7- Cortesi, L., De Matteis, E., Cirilli, C., Filieri, E., Pecchi, A., Battista, R. et al (2012). MRI before initial surgery outside of clinical trials: the real world!. European journal of radiology, 81, S21-S23.
8- Rosen, E. L., Blackwell, K. L., Baker, J. A., Soo, M. S., Bentley, R. C., Yu, D.et al. (2003). Accuracy of MRI in the detection of residual breast cancer after neoadjuvant chemotherapy. American Journal of Roentgenology, 181(5), 1275-1282.

To cite this article: Medhat M. Refaat, Ahmed A. Abou El-Soud, Manar Ahmed. The Role of MRI in Diagnosis and Management of Breast Cancer During Fertility Period. BMFJ 2022; 39 (Radiology):187-193. DOI: 10.21608/bmfj.2021.19286.1157 